\section{Falling victim to balance}

\author{
Balance: In Search of the Lost Sense \\ by Scot McCredie \\ Little, Brown: 2007.304 pp. \$24.95
}

\section{Timothy Miles}

Balance, or more precisely when people fall over because of imperfect balance, is an important issue for our ageing population. As the author of Balance, journalist Scott McCredie, points out, the social and medical costs resulting from falls are rising rapidly. One-third of people over the age of 65 and more than half of those aged 75 and over fall at least once a year, and many fall more frequently. A lot of these end up in hospital, and some never leave. Perhaps, as governments now begin to tackle the growing problem of obesity, they may also begin to think about strategies for tackling the increasing number of fall victims, which could be equally costly to society.

Scientists, pedantic folk that they are, will argue that the title of this book is misleading, given that 'balance' is not strictly a sense. Rather, balance is the outcome of an interaction of sensory signals that describe the orientation and movement of the head - which in turn stops one falling over. Thus, as the book is mainly about balance and not primarily about sensation, the reference to the "lost sense" seems rather inappropriate.

The emphasis is strongly on the workings of the vestibular system in the inner ear, with less discussion of the role of vision in balance and next to none on the important roles of sensory receptors in muscles and skin (collectively know as proprioception). This is a pity, as there is equally interesting information from both the laboratory and the clinic on the effects of disturbed proprioceptive inputs on balance and movement.

The book is wide-ranging in scope. There are many entertaining historical and anecdotal allusions ranging from Van Gogh's ear (sliced off in a futile response to intractable tinnitus or vertigo?) to The Flying Wallendas aerial balancing circus act. I was fascinated to read about the aptly but morbidly named 'lawn dart effect'. Apparently, when fighter pilots flying at low altitude accelerate horizontally at more than $1 \mathrm{~g}$, the resulting 'off-the-scale' stimulation of the vestibular system can lead to the perception that the plane is climbing. In response, the pilot pushes the joystick forward to lower his trajectory and, well, yes, that's where the name came from.

The author is clearly intrigued by alternative (read unproven) therapies in which balance/vestibular-related interventions are used to treat a range of cognitive disorders including dyslexia and even autism. In one of these, hippotherapy, children with severe neurological disorders ride horses (rather than the large

\section{Jan Stenflo}

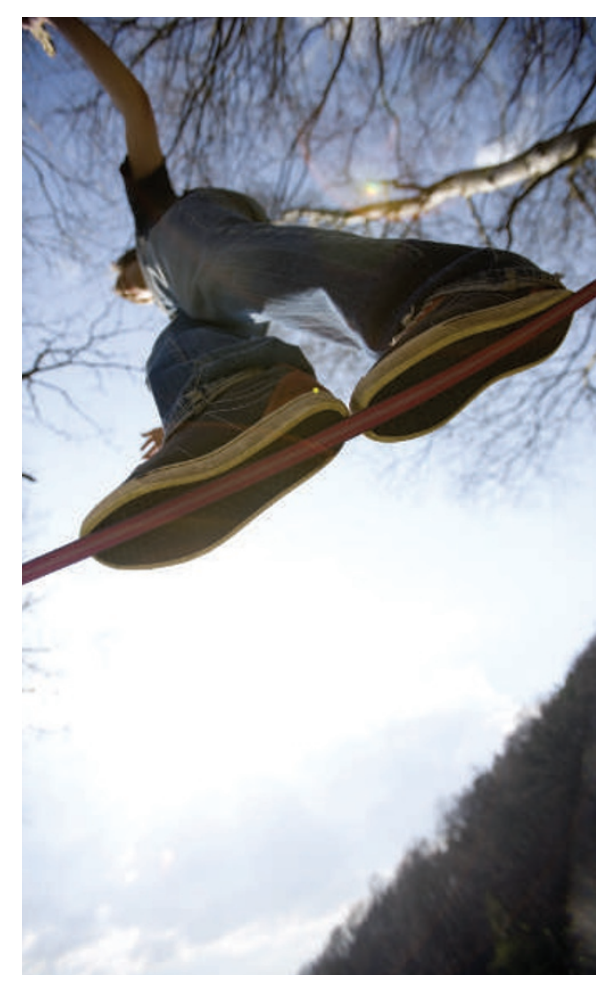

Perfect poise: balance is as important for elderly people as it is for acrobats.

African mammals) to improve their "sensory integration". The author does qualify his description of these therapies by pointing out that most of them lack proper investigation and validation, although it remains to be seen whether this disclaimer would influence parents who are desperate for an effective therapy for their autistic child, for example.

Given the breadth of material covered, there are some surprising omissions of fascinating vestibular/balance-related phenomena. Among these is the ability to "steer" subjects by stimulating their vestibular system with weak electric currents. Turning on this stimulus in blindfolded people who are walking or pushing themselves in a wheelchair causes them to veer away from their target.

There are a number of errors in the science that will cause professionals in this field to mut- ${ }^{{ }^{I}}$ ter darkly. These are not hugely consequential, but it is a pity that they escaped pre-publication screening. However, my major concern with this book is the appendix, and in particular, the exercises that are suggested therein for improving balance. The book is obviously written for lay readers rather than health-care professionals, and it seems likely that it will appeal to patients with balance disorders or their carers who are seeking more information about a particular condition and on methods for addressing it.

The strong consensus among the therapists that I consulted is that elderly or balance-compromised patients should not attempt most of the exercises recommended in the appendix without professional supervision because of the high risk of falling while doing so: in the elderly, falling has a high probability of causing serious injuries such as a hip or wrist fracture.

I found Balance to be an entertaining read, and one that should appeal to the lay reader. However, it would benefit greatly from editing by a therapist, who would either axe the appendix or add a strong disclaimer, and a neuroscientist who would sharpen the science and encourage a more even-handed view of the roles of the many sensory receptors that contribute to balance.

Timothy Miles is a professor of physiology at The University of Adelaide's Medical School, Adelaide SA 5005, Australia.

\title{
Science with flare
}

\section{The Sun Kings: The Unexpected Tragedy of Richard Carrington and the Tale of How Modern Astronomy Began \\ by Stuart Clark \\ Princeton University Press: 2007. 211 pp. \\ $\$ 24.95 / € 15.95$}

During the nineteenth century, astronomy was transformed from a mathematically oriented science preoccupied with mapping the positions and movements of celestial bodies to a physical science aiming to understand their nature and constitution. In The Sun Kings, science writer Stuart Clark offers a captivating account of this tortuous and passionately fought century-long transformation.

The tale centres around the solar astrono- mer Richard Carrington and his observations of the gigantic solar flare of 1 September 1859. Carrington, who today is little known outside the field of solar physics, happened to witness this event while drawing sunspots at his private observatory. Most solar flares can be seen only with modern spectroscopic equipment and so this white-light flare was very rare. The nearby Kew geomagnetic observatory recorded a weak magnetic disturbance lasting only 10 minutes, but exactly coincident with Carrington's flare. There was a violent geomagnetic storm 18 hours later and a worldwide display of the most magnificent aurorae, together with electric surges that disrupted telegraph communications.

Carrington's suggestion of a connection between the flare on the Sun and the geomagnetic disturbances and aurorae was largely 
dismissed and ridiculed. Even 40 years later, Lord Kelvin, then president of the Royal Society, presented a 'proof' against a solar connection in an address published in Nature. Kelvin based his proof on Maxwell's electromagnetic theory to show mathematically how it would be energetically impossible for the Sun to cause the observed disturbances in the Earth's magnetic field. Around that time Kelvin also pronounced that "there is nothing new to be discovered in physics now. All that remains is more and more precise measurements."

The case for the solar connection was finally won in 1905, in a series of debates at the Royal Astronomical Society, thanks to the statistical analysis of Walter Maunder and the physical arguments of Joseph Larmor, the new Lucasian professor of mathematics at Cambridge. Larmor invoked the recently discovered electron to make the case that beams of charged particles from the Sun disturbed Earth's magnetic field. He suggested that "investigations of this rich tapestry of particle interactions beckoned, and would distinguish the twentieth century from the nineteenth".

One of the first to advocate turning astronomy into a physical science was the eminent astronomer William Herschel in the early

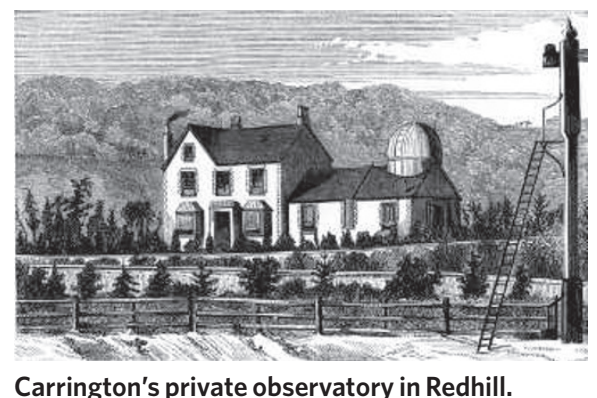

Carrington's private observatory in Redhill.

1800s. His call fell on deaf ears because it was related to another idea of his that was ridiculed, namely that the number of sunspots was statistically related to the price of wheat in England. Herschel was seeking a connection between sunspots and climate, which we now know exists. He used the price of wheat as a proxy for temperature, because there were no data on global temperature. Herschel found that when there were more sunspots, wheat was cheaper, implying warmer weather and bigger crops. Today's satellite observations show that the radiative output from the Sun is indeed higher when the sunspot number peaks.

The imprint of Carrington's 1859 flare could be seen in an ice core retrieved from Greenland in 1992. The proton beams from solar superflares produce nitrates in the Earth's atmosphere, which get deposited in the ice. Of all the flares of the past 500 years, the 1859 flare was one of the most powerful.

The most revolutionary tool for the physical exploration of stars was spectroscopy. Dark lines in the solar spectrum were noticed by William Wollaston in 1801 and rediscovered by Joseph von Fraunhofer in 1814, who later mapped these lines in much greater detail with his invention, the diffraction grating. Robert Bunsen and Gustav Kirchhoff showed that these absorption lines were the fingerprints of chemical elements - despite the famous French philosopher's Auguste Comte 1835 pronouncement that we would never know how to study the chemical composition of the stars.

Stuart Clark weaves all these events and ideas together in a fascinating tapestry. The account is accurate while being non-technical, and is suited for anyone with a general interest in the history of science. Clark's engaging writing style conveys the passion, intrigues and captivating life stories of the main players. It is a gripping tale of the birth of modern astronomy. Jan Stenflo is professor of astronomy at the Institute of Astronomy, 8092 Zurich, Switzerland.

\section{A greener education}

\section{Degrees that Matter: Climate Change and the University \\ by Ann Rappaport and Sarah Hammond Creighton \\ MIT Press: 2007.376 pp. \$24.95, £15.95}

\section{Peter Hopkinson}

Globally, higher education is a major service sector employing hundreds of thousands of people, educating millions of students, carrying out billions of pounds' worth of research and running huge campuses. These activities consume large amounts of fossil fuel and hence release significant amounts of carbon dioxide and other greenhouse gases. The central observation from Degrees that Matter is that universities are in a unique position to offer leadership on climate change and carbon emissions through their educational, research and wider roles in society.

This book is a case study of the attempts made at Tufts University, Massachusetts, to reduce carbon emissions through the Tufts Climate Initiative, a 15-year programme that began in 1991. The book was written by the initiative's directors and provides an exhaustive 330-page review and report on the actions taken to reduce carbon emissions at the university. Examples of initiatives include switch-off campaigns, procuring where possible renewable energy, promoting greener forms of travel and working with students and faculty mem- bers to avoid energy waste. The good news is that the book has a wealth of ideas, recommendations and guidance.

For anyone who is responsible for energy or carbon management in a university, or is seeking to engage students and faculty in climate change, this book is compulsory reading. The chapters on personal action and climate change in the classroom are borne out of extensive experimentation and experience at Tufts and provide real stimulus for action within highereducation establishments.

The bad news is that despite the intense programme, carbon emissions at Tufts — both net and normalized - seem to have increased over time. The university as a whole has become more energy intensive, with the consequence that it will not meet its Kyoto target. This should, however, be set against other higher-education institutions, where the rate of increase over similar time periods is much greater and the reversal of trends, if at all, much slower. A large part of the increase is due to growing demands from personal equipment.

The picture may be even worse because the data do not take into account carbon emissions from staff, students and visitors commuting, or from business travel, which is widely accepted as having increased dramatically since 1990. The issue of travel is not given a great deal of attention in the book, although it is a source of considerable tension within any university seeking to both promote 'internationalization' and reduce carbon emissions.

The one main weakness of the book is that there is relatively little empirical data on emissions sources, and little evaluation of the impact that the different initiatives at Tufts have had on emissions. The authors acknowledge the problems of measuring energy use and emissions, and argue that data collection, monitoring and reporting are crucial to carbon management and reduction. This is not an issue only at Tufts but is typical of most highereduction institutes where, historically, environmental-performance data have not been a priority, with a consequent lack of investment in systems for measuring and recording energy consumption .

With climate change and carbon emissions high on the political and educational agenda, the book is a timely reminder to the highereducation sector that it needs to attend to its own performance and reputation in this area. The evidence from Tufts shows the difficulties of reducing carbon emissions, but the Tufts Climate Initiative does provide a very good plan of action. The book's central message is that if universities wish to demonstrate practical leadership on reducing carbon emissions, there is a need for much greater action and progress than is currently found in most higher-education institutions.

Peter Hopkinson is director of education for sustainable development at the University of Bradford, West Yorkshire BD7 1DP, UK, and co-director of Higher Education Environmental Performance Improvement (HEEPI). 